\title{
ON MAXIMAL TORSION RADICALS. III
}

\author{
JOHN A. BEACHY
}

\section{ABSTRACT. Maximal torsion radicals of a strongly semiprime ring} correspond to minimal prime ideals and can be used to characterize both strongly semiprime and semiprime Goldie rings.

Let $R$ be an associative ring with identity and let $R$-Mod denote the category of unital left $R$-modules. A subfunctor $\sigma$ of the identity 1 on $R$-Mod is called a torsion preradical if $\sigma\left(M^{\prime}\right)=M^{\prime} \cap \sigma(M)$ for all $R$-submodules $M^{\prime}$ $\subseteq M$, and a torsion radical if, in addition, $\sigma(M / \sigma(M))=0$ for all $R$-modules $M$. A torsion radical $\sigma$ is called maximal if it is proper $(\sigma \neq 1)$ and maximal with respect to the relation $\leq$, where $\tau \leq \sigma$ if $\tau(M) \subseteq \sigma(M)$ for all $R$-modules $M$. For $R^{M}$ the largest torsion radical $\sigma$ such that $\sigma(M)=0$ will be denoted $\operatorname{rad}_{E(M)}$, where $E(M)$ is the injective envelope of $M$. It is a well-known result that for a commutative Noetherian ring $R$ there is a one-to-one correspondence between maximal torsion radicals of $R$-Mod and minimal prime ideals of $R$. This correspondence was recently extended by the author [2] to rings with Krull dimension on either the left or right. This paper shows that the correspondence holds for semiprime rings which satisfy a very weak form of d.c.c. on left annihilators, which holds in particular for any semiprime left Goldie ring.

Following the terminology of Handelman and Lawrence [6], a ring $R$ will be called left strongly prime if for each $0 \neq b \in R$ there exist $r_{1}, r_{2}$, $\ldots, r_{n} \in R$ such that $a r_{i} b=0$ for all $i$ implies $a=0$, for any $a \in R$. Such rings are just the left absolutely torsion free rings introduced by Rubin [7], who characterized them as rings $R$ such that $R$-Mod has a maximal torsion radical which contains all proper torsion preradicals. Viola-Prioli [8] showed that a ring is left strongly prime if and only if every nonzero left ideal is cofaithful. (A module ${ }_{R} M$ is cofaithful if there exists an embedding $0 \rightarrow R \rightarrow M^{n}$ of $R$ in a finite direct sum of copies of $M$, or equivalently, if there exists a finite subset of $M$ whose left annihilator is zero.) The notion can be extended as in [4] by calling a ring left strongly semiprime if it is semiprime and every faithful left ideal is cofaithful, the latter part of the condition being a weak form of d.c.c. on left annihilators. An equivalent condition is that every essential left ideal is cofaithful [5].

Received by the editors August 5, 1974 .

AMS (MOS) subject classifications (1970). Primary 16A08, 16A12, 18E40.

Key words and phrases. Semiprime Goldie ring, strongly semiprime ring, maximal torsion radical. 
Lemma (1). If every faithful left ideal of $R$ is cofaithful, then every proper right annihilator ideal of $R$ is contained in a maximal right annibilator ideal.

Proof. An ideal $A$ is called a right annihilator if $A \neq 0$ and $A=n(S)=$ $\{r \in R \mid S r=0\}$ for some subset $S \subseteq R$. (The left annihilator $\ell(S)$ is defined similarly.) If $A$ is a proper right annihilator ideal of $R$, let $\left\{A_{a}\right\}_{a \in I}$ be any chain of proper right annihilator ideals each of which contains $A$. If $\ell\left(\bigcup_{a \in I} A_{\alpha}\right)=0$, then $\bigcup_{a \in I} A_{\alpha}$ is faithful and therefore cofaithful by assumption, so there exist $a_{1}, \ldots, a_{n} \in \bigcup_{a \in I} A_{a}$ such that $\ell\left(a_{1}, \ldots, a_{n}\right)=0$. Since $\left\{A_{\alpha}\right\}_{\alpha \in I}$ is a chain, $a_{1}, \ldots, a_{n} \in A_{a}$ for some $\alpha \in I$, and then $\mathcal{l}\left(A_{\alpha}\right)=0$ and $A_{a}=R$, a contradiction. Since $\ell\left(\bigcup_{a \in I} A_{a}\right) \neq 0, r \ell\left(\bigcup_{a \in I} A_{a}\right)$ is a proper right annihilator ideal and is a least upper bound for the chain $\left\{A_{a}\right\}_{a \in I}$. By Zorn's lemma there is a maximal element in the set of proper right annihilator ideals containing $A$.

Theorem (2). If $R$ is a left strongly semiprime ring, then every torsion radical of $R$-Mod is contained in a maximal torsion radical, and there is a one-to-one correspondence between maximal torsion radicals of $R$-Mod and minimal prime ideals of $R$.

Proof. Let $K \neq R$ be a nonzero torsion ideal $(K=\sigma(R)$ for some torsion radical $\sigma$ ) of the left strongly semiprime ring $R$. Then $K$ is not cofaithful since $R \subseteq K^{n}$ would imply $K=R$. Since $R$ is strongly semiprime, $K$ is not faithful and hence $\ell(K) \neq 0$, so $n \ell(K)$ is a proper right annihilator ideal containing $K$. By Lemma 1 there is a maximal right annihilator ideal $P \cdot$ which contains $K$, with $\ell(P) \subseteq \ell(K)$. Since $R$ is semiprime, $\ell(K) \cap K=0$ and $r(P)=$ $\ell(P)$, so that $P$ is the annihilator of a submodule of $R / K$ isomorphic to $n(P)$, and therefore $\operatorname{rad}_{E(R / P)} \geq \operatorname{rad}_{E(R / K)}$. Since any maximal annihilator is a minimal prime, this shows that for any torsion ideal $K$ there is a minimal prime ideal $P$ such that $\operatorname{rad}_{E(R / P)} \geq \operatorname{rad}_{E(R / K)^{\circ}}$. (If $R$ is prime, then 0 is a minimal prime, and if not, then as above there is a maximal annihilator $P$

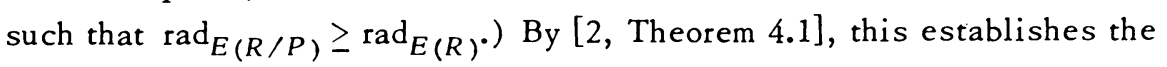
theorem.

The proof of Theorem 2 shows that every maximal torsion radical $\mu$ is of the form $\operatorname{rad}_{E(R / P)}$ for some maximal right annihilator ideal $P$, and since $R$ is semiprime, $P$ is a left annihilator; the proof of [1, Theorem 2.2] shows that since $P$ is a closed ideal of a semiprime ring, $\operatorname{Hom}_{R}(P, E(R / P))=0$ and therefore $P=\mu(R)$. As a consequence of Theorem 2, the minimal primes of $R$ are just the maximal right annihilator ideals. Moreover, there are only finitely many minimal primes (by the following theorem) generalizing the wellknown result in the special case of semiprime left Goldie rings.

Theorem (3). The ring $R$ is left strongly semiprime $\Leftrightarrow$ every proper tor- 
sion preradical of $R-\operatorname{Mod}$ is contained in a maximal torsion radical and $R$-Mod has only finitely many maximal torsion radicals.

Proof. $(\Longrightarrow)$ If $\tau$ is any proper torsion preradical, then $\tau(R)$ is not cofaithful, so as in the proof of Theorem 2 there is a maximal right annihilator ideal $P$ with $r(P) \cap \tau(R)=0$. Then $\tau(R / P)=0$ since $r(\tau(P))=0$ and $P$ can be embedded in a direct product of copies of $n(P)$. This implies that $\tau \leq$ $\operatorname{rad}_{E(R / P)}$, so every proper torsion preradical is contained in a maximal torsion radical. If $A_{i} \neq A_{j}$ are minimal left annihilator ideals of $R$, then $A_{i} \cap$ $A_{j}=\ell\left(n\left(A_{i}\right) \cup n\left(A_{j}\right)\right)$, which forces $A_{i} \cap A_{j}=0$ and thus $A_{i} A_{j}=0$ since $R$ is semiprime. From this it follows that the sum in $R$ of all minimal left annihilator ideals must be a direct sum, and then since $R$ is left strongly semiprime, it cannot contain an infinite direct sum of ideals [4, Proposition 1.3], so the sum is finite. Thus there are only finitely many maximal right annihio lator ideals $P_{1}, \ldots, P_{n}$ which determine maximal torsion radicals $\mu_{1}, \ldots$, $\mu_{n}$ with $\mu_{i}(R)=P_{i}$ for $i=1, \ldots, n$.

$\Leftrightarrow$ Let $\mu$ be any maximal torsion radical of $R$-Mod, with $\mu(R)=P$, let $A / P$ be any nonzero left ideal of $R / P$, and let $\tau$ be the smallest torsion preradical such that $\tau(A / P)=A / P$ and $\tau \geq \mu$. Then $\tau=1$ since $\tau \neq \mu$ and by assumption any proper torsion preradical must be contained in a maximal torsion radical. Since $\mu(E(R / P))=0, \tau(E(R / P))$ must be the sum of all homomorphic images of $A / P$, and so $A / P$ generates $E(R / P)$, which implies that $A / P$ is cofaithful as an $R / P$-module. Thus $R / P$ is strongly prime since every nonzero left ideal is cofaithful.

Let $K=\bigcap_{i=1}^{n} \mu_{i}(R)$, where the intersection runs over all maximal torsion radicals, and let $\sigma$ be the smallest torsion preradical such that $\sigma(R / K)=$ $R / K$. Then $\sigma\left(R / \mu_{i}(R)\right) \neq 0$ since $R / \mu_{i}(R)$ is a homomorphic image of $R / K$, which implies $\sigma \underline{\not} \mu_{i}$ for all $i$, and therefore $\sigma=1$. It follows that $R / K$ is cofaithful, and so $K=0$ and $R$ is a finite subdirect product of strongly prime rings, which shows by [5, Proposition 5] that $R$ is a strongly semiprime ring.

The final theorem, when combined with Theorem 3, gives a characterization of semiprime Goldie rings which is stated in purely torsion theoretic language. Recall that a torsion radical $\sigma$ is called perfect if the quotient functor defined by $\sigma$ is naturally isomorphic to $Q_{\sigma}(R) \otimes_{R}$-, where $Q_{\sigma}(R)$ is the corresponding ring of quotients. If the ring $R / \sigma(R)$ has zero singular ideal, then the following are equivalent: (i) $\sigma$ is perfect; (ii) $R / \sigma(R)$ has finite uniform dimension; (iii) $Q_{\sigma}(R)$ is semisimple Artinian [3, Proposition 3.9]. Furthermore, a semiprime ring is Goldie if and only if it has finite uniform dimension and zero singular ideal.

Theorem (4). $R$ is a semiprime left Goldie ring $\Leftrightarrow R$ is left strongly 
semiprime and every maximal torsion radical of $R-\operatorname{Mod}$ is perfect.

Proof. It can be shown that if $R$ is strongly semiprime, then with the notation of Theorem 3, $R$ is an essential $R$-submodule of the direct product $\Pi_{i=1}^{n} R / P_{i}$, just as in [5, Proposition 9$]$, and so $E(R) \simeq \bigoplus_{i=1}^{n} E\left(R / P_{i}\right)$. Since $P_{i}$ is a torsion ideal, the $R$-injective envelope and $R / P_{i}$-injective envelope coincide, and thus $Q_{\max }(R) \simeq \Pi_{i=1}^{n} Q_{\max }\left(R / P_{i}\right)$. (The complete ring of quotients $Q_{\mathrm{max}}(R)$ coincides with $E(R)$ since $R$ has zero singular ideal, as shown by [5, Proposition 6]; the same is true of $R / P_{i}$. ) From the remarks preceding the theorem, it follows immediately that $R$ is Goldie if and only if $Q_{\max }(R)$ is semisimple Artinian, and this occurs if and only if each maximal torsion radical of $R$-Mod is perfect.

\section{REFERENCES}

1. John A. Beachy, On maximal torsion radicals, Canad. J. Math. 25 (1973), $712-726$.

2. - On maximal torsion radicals. II, Canad. J. Math. 27 (1975), 115-120.

3. - Perfect quotient functors, Comm. Algebra 2 (1974), 403-427.

4. John A. Beachy and William D. Blair, Rings whose faithful left ideals are cofaithful, Pacific J. Math. (to appear).

5. D. Handelman, Strongly semiprime rings, Pacific J. Math. (to appear).

6. D. Handelman and J. Lawrence, Strongly prime rings, Trans. Amer. Math. Soc. 211 (1975), 209-223.

7. Robert A. Rubin, Absolutely torsion-free rings, Pacific J. Math. 46 (1973), 503-514.

8. Jorge Viola-Prioli, On absolutely torsion free rings and kernel functors, Thesis, New Brunswick, N. J., Rutgers University, 1973.

DEPARTMENT OF MATHEMATICAL SCIENCES, NORTHERN ILLINOIS UNIVERSITY, DE KALB, ILLINOIS 60115 